\title{
Research of Cellular Automata Traffic Flow Model for Variable Traffic Flow Density
}

\author{
Zhang Jian-hua, Jiang Tao, Wang Sheng-an, Ma Jia-wei \\ XuZhou Institute of Technology \\ Xuzhou, Jiangsu, 221111, China \\ E-mail: 1044042112@qq.com
}

\begin{abstract}
Freeway traffic flow and safety are the main subjects of the current highway traffic operation in an intelligent transportation system. The traffic flow model and traffic safety evaluation model were studied in this paper to analysis the traffic operation under a new traffic rule of variable traffic flow density. The classic car-following model is used to describe the traffic flow under the intelligent traffic system, and the variable traffic flow density is derived according to the constraints of the left overtaking. The corresponding interval values of average speed discrete degree are obtained to score the traffic safety degree. Then cellular automata is used to simulate the model, finding that traffic flow remains unchanged, while the safety of the traffic increases.
\end{abstract}

Keywords-traffic flow; traffic safety; traffic flow density; traffic flow velocity; cellular automata

\section{INTRODUCTION}

Intelligent transportation systems (ITS) is the future research of the transport system, which applies the data communication and transmission technology, electronic sensor technology, control engineering technology to the entire surface traffic system to establish effectively an integrated transport management system with advantages as large scale, real-time, accurate, and efficient.

Research on traffic flow includes car following theory, fluid dynamics simulation, vehicles queuing theory, Cellular Automata (CA) and so forth. Kinetic methods and mathematical model are used to explore the following car's state when the car can only drive in a single lane in car following thoery[1]. Fluid dynamics simulation is proposed by British scholar Lightill and Whitham[2]. Traffic flow is regarded as a kind of fluid and the law of the traffic flow is studied under the conditions of a high traffic density on a long road tunnel. Vehicles queuing mainly discusses the vehicle queue length and the chain reaction[3], which can be adapted to the different sections and the intersections of a road. Cellular automata is mainly used to simulate a variety of traffic flows through the movement of the unit cell. At present, it is combined with many other theories and has been used in many aspects[4,5].

Traffic safety is mainly to establish a safety evaluation system which includes a comprehensive analysis of vehicle data, grading within a certain time. Literature [6] introduced multiple heterogeneous data sources for highway traffic safety assessment. Literature [7] evaluated the traffic safety of highway based on the traffic speed.
Literature [8] researched the traffic flow model by Agent technology, genetic algorithms, etc.

\section{TRAFFIC MODEL IN INTELLIGENT TRANSPORT SYSTEM}

\section{A. Steady and Balanced Traffic Density}

When the distance between two vehicles, denoted as $x_{j . j-1}$, is longer, the attractive force during the overtaking, denoted as $F$, is smaller. While the difference of driving speed between the two vehicles, denoted as $u_{j, j-1}$, is bigger, the attractive force will be greater. Therefore, the attractive force can be expressed as ${ }^{[9]}$ :

$$
F=k u_{j, j-1} / x_{j . j-1}
$$

where $k$ is a positive proportionality factor. $x_{j . j-1}$ and $u_{j, j-1}$ can be concluded according to the distance and speed between two vehicles as following:

$$
x_{j, j-1}=x_{j}-x_{j-1}, u_{j, j-1}=\frac{d x_{j}}{d t}-\frac{d x_{j-1}}{d t}
$$

Then the circular motion law can be expressed as

$$
M \frac{d^{2} x_{j}(t)}{d t^{2}}=k \frac{u_{j, j-1}}{x_{j . j-1}}=k \frac{x_{j, j-1}^{\prime}}{x_{j . j-1}}
$$

where $M$ is the weight of the vehicle.

The traffic flow velocity can be derived by integrating the above equation as following:

$$
u=\frac{k}{M} \ln \left(\left|x_{j}-x_{j-1}\right|\right)
$$

As for the steady and smooth traffic flow, the distances between the vehicles are the same, marked as $d$. Then the traffic density is given by

$$
\rho=\frac{1}{L+d}
$$

where $L$ is the length of the vehicle.

When there are relatively fewer vehicles, driving speed of the vehicle and the traffic density can reach the maximum $u_{\max }$ and critical density $\rho_{c}$. Similarly, the traffic density also has a maximum value $\rho_{\max }$, and the velocity at this moment is $u=0$. Therefore, during one overtaking, the change of traffic flow speed can be expressed as the function of traffic density as follows 


$$
u(\rho)= \begin{cases}u_{\max }, & 0 \leq \rho \leq \rho_{c} \\ u_{\max } \frac{\ln \left(\rho_{\max } / \rho\right)}{\ln \left(\rho_{\max } / \rho_{c}\right)}, & \rho_{c} \leq \rho \leq \rho_{\max }\end{cases}
$$

The function of the traffic flow can be concluded according to the basic theory of traffic flow

$$
q=u \rho
$$

\section{B. Variable Traffic Flow Density}

However, the traffic density is not necessarily a constant value in our daily life. If the whole highway is taken as the study object, and the traffic inflow and outflow are the same, namely,

$$
Q_{\text {in }}(t)=Q_{\text {out }}(t)
$$

Thus, the traffic density can be expressed as the function related to the rate of overtaking and changing lanes. The rate of changing lanes is marked as $k(t)$, which will not change because of the different lanes, but change with the time. Reference [3] gives the rate of times of overtaking through simulations, and measures the numbers of overtaking and ciphers out the rate of times of overtaking and the overtaking rate. Lane changing only happens on the neighboring lanes, and each lane-changing on the left must be followed by a lane-changing to the right according to the rule of driving on the right. Then, traffic density must conform to the following relation:

$$
\begin{gathered}
\rho_{i}(t+1)=\rho_{i}(t)-k(t)\left(\rho_{i}(t)-\rho_{i-1}(t)\right) \\
-k(t)\left(\rho_{i}(t)-\rho_{i-1}(t)\right)
\end{gathered}
$$

Here, $\rho_{i}(t)$ is used to indicate the traffic density on the lane $i$ at moment $t, i=2,3, \ldots, n-1$.

When $i=1$, the traffic density is given by

$$
\rho_{1}(t+1)=\rho_{1}(t)-k(t)\left(\rho_{1}(t)-\rho_{2}(t)\right)
$$

When $i=n$, the traffic density is given by

$$
\rho_{n}(t+1)=\rho_{n}(t)-k(t)\left(\rho_{n}(t)-\rho_{n-1}(t)\right)
$$

As for the double-lane highway, overtaking needs to change from the right lane to the left and then change from the left lane back to the right, and the lane-changing can be expressed with a matrix, as follows:

$$
\rho(t+1)=\left[\begin{array}{cc}
1-k(t) & k(t) \\
k(t) & 1-k(t)
\end{array}\right] \rho(t)
$$

The factors in the matrix represent the probability of changing lanes. According to the rule of driving on the right, it is necessary to give a limitation to the times of changing lanes. The total number on the left lane is 0 , if the overtaking vehicle speed up to the maximum of the left lane and then run at a constant speed, we mark the speed limit on the left lane as $u_{\text {max }}^{\prime}\left(u_{\text {max }}^{\prime} \geq u_{\max }\right)$ then:

$$
\sum u_{\text {max }}^{\prime} \rho_{2}(t)=0
$$

When we decide the initial value of traffic density, we may use the steady and smooth traffic flow as the initial value on the right lane, and infer the changes of the traffic density during the whole process according to it.

In contrast with the steady and smooth traffic flow, the traffic density here includes the overtaking limits while the relation between traffic velocity and traffic density remain the same. So, we can also infer the traffic velocity according to the equation (12). Finally, we define the traffic density on the whole way, taking the rule of driving on the right and overtaking on two lanes into consideration. The equation is as follows:

$$
q= \begin{cases}\rho_{1}(t) u_{\max }+\rho_{2}(t) u_{\text {max }}^{\prime} & , 0 \leq \rho \leq \rho_{c} \\ \frac{\left[u_{\max } \ln \frac{\rho_{\text {max }}}{\rho_{1}(t)}+u_{\text {max }}^{\prime} \ln \frac{\rho_{\max }}{\rho_{2}(t)}\right]}{\ln \left(\rho_{\max } / \rho_{c}\right)}, \rho_{c} \leq \rho \leq \rho_{\max }\end{cases}
$$

With the help of the references [10], we set the speed limit as $u_{\max }=100 \mathrm{~km} / \mathrm{h}$, the average length of vehicles as $L=4 m$, the greatest traffic density as $\rho_{\max }=1 \mathrm{pcu} \cdot \mathrm{km}^{-1}$, the critical traffic density as $\rho_{c}=0.25 \mathrm{pcu} \cdot \mathrm{km}^{-1}$. Substitute them into the above equation and we will get the changes of traffic flow on a highway in simulation. The driving speed, traffic density and traffic flow value can be indicated as the following tables.

TABLE I. SIMULATION VALUES OF TRAFFIC FLOW, TRAFFIC VELOCITY AND TRAFFIC DENSITY

\begin{tabular}{c|c|c|c|c|c}
\hline $\begin{array}{c}\text { Traffic } \\
\text { density }\end{array}$ & 0.1 & 0.2 & 0.3 & 0.4 & 0.5 \\
\hline $\begin{array}{c}\text { Traffic } \\
\text { velocit } \\
\text { y }\end{array}$ & 100 & 100 & 86.84 & 66.09 & 50 \\
$\begin{array}{c}\text { Traffic } \\
\text { flow }\end{array}$ & 10 & 20 & 26.25 & 26.43 & 25 \\
$\begin{array}{c}\text { Traffic } \\
\text { density }\end{array}$ & 0.6 & 0.7 & 0.8 & 0.9 & 1 \\
$\begin{array}{c}\text { Traffic } \\
\text { velocit } \\
\text { y }\end{array}$ & 36.84 & 25.72 & 16.09 & 7.60 & 0 \\
$\begin{array}{c}\text { Traffic } \\
\text { flow }\end{array}$ & 22.10 & 18.01 & 12.87 & 6.84 & 0 \\
\hline
\end{tabular}

In order to indicate the process of the changes, we alter the critical density, the maximum speed, and get the changing tendency of the traffic velocity and traffic flow under several conditions. The change function is demonstrated as the following figures.

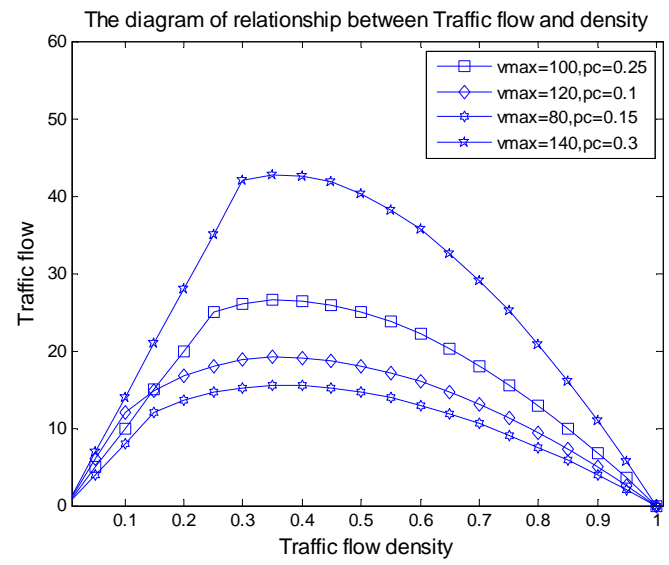

Figure 1. Relationship between traffic flow and density

It can be seen that driving speed remains constant before the state of critical density and the traffic flow goes up like a rocket, then the driving speed becomes slow down, while the traffic flow decreases after a period of 
increasing. When the traffic density tends to maximum, both the traffic velocity and the traffic flow tend to become zero, which is consistent with the situations in our daily life.

The speed limit on the overtaking lane is set as $u_{\text {max }}^{\prime}=120 \mathrm{~km} / \mathrm{h}$, and the steady and smooth traffic flow is adopted as the initial value of the density on the right lane. After setting the traffic density on the lanes, we can get the values of traffic flow, traffic velocity and traffic density. And a change function of traffic flow is demonstrated in the following diagram.

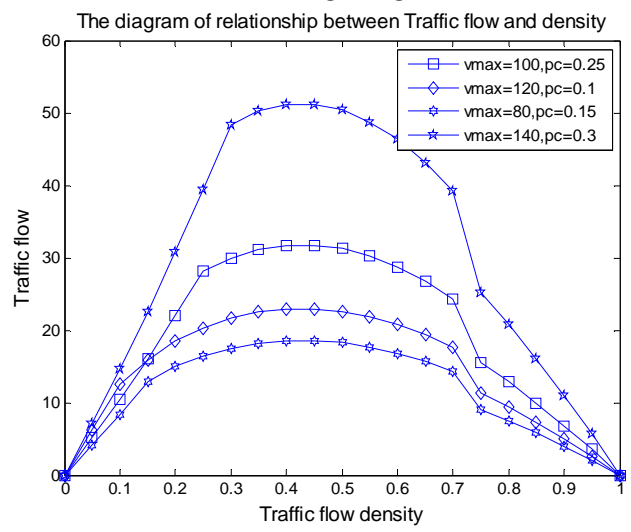

Figure 2. Relationship between traffic flow and density

\section{Traffic Safety Model on average speed discrete degree $e^{[11]}$}

The traffic performance of a vehicle with others is considered in the freeway. A vehicle is inclined to overtake another vehicle when it has a faster speed. Less the speed a vehicle is, less the percentage of overtaking the vehicle ahead is, more percentage of being overtook by the vehicle rear is. So reducing the discrete degree between the vehicles can reduce the possibility of an accident.

The point speed of a vehicle at the section observation is used to express arithmetic mean value $\bar{v}$,

$$
\bar{v}=\sum_{i=1}^{n} v_{i} / n
$$

In order to study the distribution of the traffic flow, speed discrete degree is defined firstly as

$$
S D=\sqrt{\sum_{i=1}^{n}\left(v_{i}-\bar{v}\right)^{2} /(n-1)}
$$

In a certain time $t$, the vehicle gets through the section $i$ of the freeway. With the ahead and rear sections with the same distance $L$ to the section $i$ as boundaries, there are two segments, respectively, $R_{1}$ and $R_{2}$.

When driving distance is very short, average speed of a vehicle is equal to the instantaneous velocity. According to all the vehicles' speeds $v_{i}$ in the segment $R_{1}$ and $R_{2}$, the point speed of a vehicle through segment $i$ at the time $t$ and the vehicle numbers $n_{1}, n_{2}$ in segments $R_{1}$ and $R_{2}$, the average speed discrete degree (ASD) is define $\mathrm{d}$ as

$$
A S D=\sqrt{\sum_{i=1}^{n_{1}+n_{2}}\left(v_{i}-v\right)^{2} /\left(n_{1}+n_{2}\right)}
$$

According to the average speed of China freeway, the speed discrete degree, regression model of speed discrete degree and accident times of a hundred million vehicles per kilometers $\left(n \cdot 10^{-9} \cdot v^{-1} \cdot \mathrm{km}^{-1}\right)$ are analyzed as following

$$
A R=9.5839 e^{0.0553 S D}
$$

In order to show the relation between the speed dispersion and the number of accidents, the curve of the speed dispersion is demonstrated as follows.

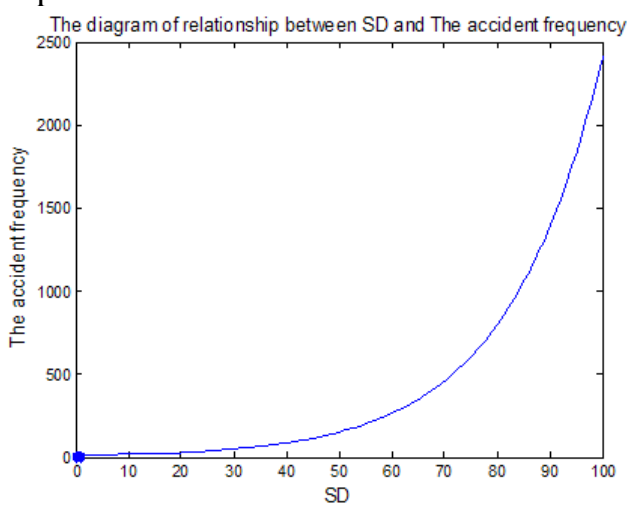

Figure 3. Relation between speed dispersion and accidents frequency

From the above figure, it can be seen that the number of accidents becomes bigger with the increase of speed dispersion, which complies with the practical situations. The bigger the number of accidents becomes, the lower the safety degree will be.

The number of corresponding accidents at different levels given by FHWA-RD-99-174 is given in the table II.

TABLE II. STORY RATES CORRESPONDING TO DIFFERENT SECURITY

\begin{tabular}{ccc}
\hline $\begin{array}{c}\text { security } \\
\text { degrees }\end{array}$ & security & times $\cdot 10^{-9} \cdot \mathrm{veh}^{-1} \cdot \mathrm{km}^{-1}$ \\
\hline 1 & govels & {$[0,46]$} \\
2 & moderate & {$[46,144]$} \\
3 & bad & {$[144,276]$} \\
\hline
\end{tabular}

TABLE III. SPEED STANDARD DEVIATION CORRESPONDING TO DIFFERENT SECURITY LEVELS

\begin{tabular}{ccc}
\hline $\begin{array}{c}\text { security } \\
\text { degrees }\end{array}$ & $\begin{array}{c}\text { security } \\
\text { levels }\end{array}$ & Speed standard deviation \\
\hline 1 & good & {$[0,28.36]$} \\
2 & moderate & {$[28.36,49.60]$} \\
3 & bad & {$[49.60,76]$} \\
\hline security & security & speed dispersion \\
degrees & levels & {$\left[(\mu-v), \sqrt{804.29+(\mu-v)^{2}}\right]$} \\
\hline 1 & good & \\
2 & moderate & {$\left[\sqrt{804.29+(\mu-v)^{2}}, \sqrt{2401+(\mu-v)^{2}}\right]$} \\
3 & bad & {$\left[\sqrt{2401+(\mu-v)^{2}}, \sqrt{3691.77+(\mu-v)^{2}}\right]$} \\
\hline
\end{tabular}

Substituting the number of accidents into the equation of speed dispersion and the number of accidents, we can get the corresponding speed standard deviations as 0 , 28.36, 49.60, 79. The speed standard deviations of 
different security levels can be derived as Table III.

In order to score the safety degrees, we need to score the corresponding security levels of the speed dispersion as follows:

When $(\mu-v)<A S D<\sqrt{804.29+(\mu-v)^{2}}$, the score of the speed dispersion is between $80-100$.

When $\sqrt{804.29+(\mu-v)^{2}}<A S D<\sqrt{2401+(\mu-v)^{2}}$, the score of the speed dispersion is between 60-80.

When $\sqrt{2401+(\mu-v)^{2}}<A S D<\sqrt{3691.77+(\mu-v)^{2}}$, the score of the speed dispersion is between 0-60.

\section{NUMERICAL SIMULATION BASED ON CELLULAR AUTOMATA}

\section{A. Simulation Results of Traffic flow}

By the rules of the intelligent systems set above, the situations by Matlab is given according to the prevailing road conditions. Since cellular automata are very widely used to simulate a variety of discrete and random natural phenomena, we introduce cellular automata to imitate actual vehicle simulation in the specific simulation. Cellular automata includes cellular space, cellular state, state neighbors and spatial movement rules ${ }^{[11]}$.

The evolution rules of the CA can be described as

$$
f: C C^{Y} \rightarrow C C: \bigcap_{j \in N C_{i}(t)} \sigma_{j}(t) \mapsto \sigma_{i}(t+1)
$$

where $Y$ is the number of $N C$. $N C_{i}(t)$ is the state of $i$-th adjacent cellular at time $t$, and $\sigma_{j}(t)$ is its parameters.

Sine the neighbor $C C^{Y}$ of each CA may cast a light on status numbers, total structure of CA is expressed as

$$
C(t)=\bigcap_{j \in C S} \sigma_{j}(t)
$$

Applying part-changing rules to all CA in the net, then following cast could be calculated by polynomial

$$
G: C C^{C S} \rightarrow C C^{C S}: C(t) \mapsto C(t+1)
$$

Thus the whole structure of CA can be obtained at any time $t$. We get large dates and show them as follows.

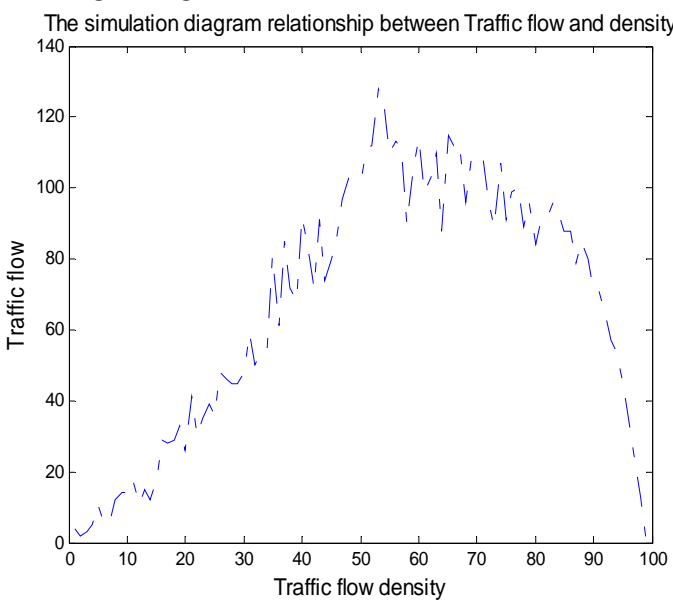

Figure 4. Simulation results of traffic flow

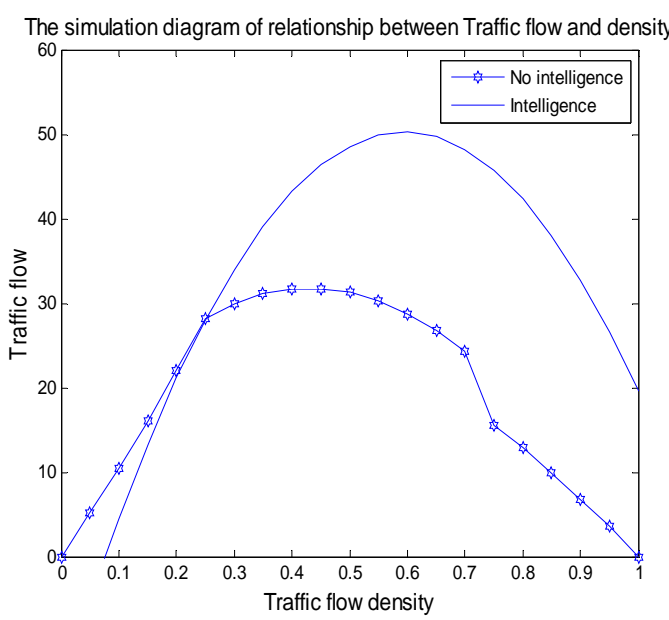

Figure 5. Simulation between traffic flow and density

\section{B. Numerical Simulation of the Traffic Safety}

Based on the above ASD model, numerical simulations of cellular automata is used to obtain the traffic volume and speed of evaluation, vehicle safety in the intelligent systems ${ }^{[12]}$.

Because vehicle safety is a priority in the intelligent systems, that is, all actions of a vehicle are carried out in order to obtain security. But this can only be guaranteed when the safety of scores is greater than 60 without considering unexpected situations, such as a flat tire, snow and slippery nature disasters. Then we introduce the correction factor in the traffic flow to correct the impact velocity dispersion and vehicle speed on the pitch with the appropriate degree of safety. Actual vehicle driving safety $Z^{\prime}$ under intelligent control system can be expressed as

$$
Z^{\prime}=C\left(w_{v} v+w_{d} d\right)+b
$$

From non-intelligent system control schematic diagram, it can be found that the change of the safety is decreased with increasing traffic density, and vehicle safety has a period of stability in the region with the safety degree greater than 60 . The safety degree is stabilized to 80 with the traffic density, then the correction coefficient is expressed as

$$
C=\frac{80}{60}=\frac{4}{3}
$$

Therefore, actual vehicle driving safety in the intelligent system can be expressed as

$$
Z^{\prime}=\frac{4}{3}\left(w_{v} v+w_{d} d\right)+b
$$

To compare the safety between intelligent and non-intelligent control system, the safety degree of the two cases are put together and the comparisons are as follows.

It can be clearly seen from the figure, the vehicle running under the security control of the intelligent system is significantly lower than one with no intelligent system safety. This also demonstrates the model is efficient. 


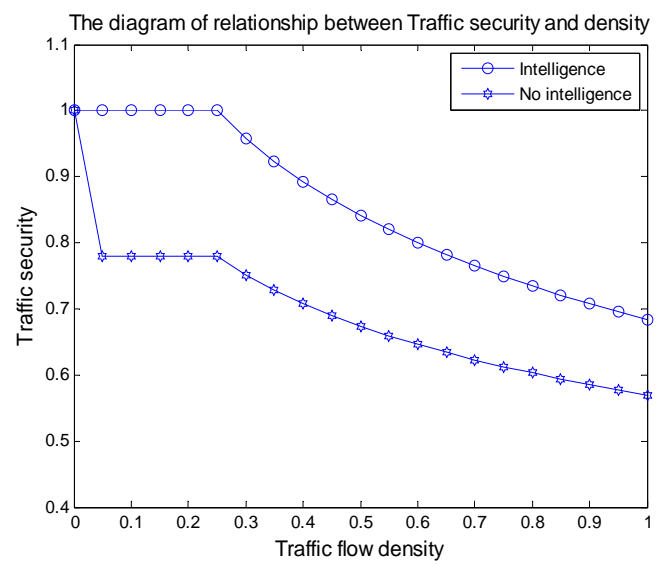

Figure 6. Simulation results of traffic safety

\section{CONCLUSIONS}

The traffic flow model and traffic safety evaluation model were studied in this paper. Using car-following model as the basic model, we studied the changes of highway traffic flow under balanced traffic density and variable density. In both cases, traffic flow trends to be the same with the roughly maximal traffic density. For security issues, model of average speed discrete degree is used to score the safety. The higher the velocity dispersion is, the bigger the number of accidents is, and the lower the degree of safety is. Finally, we made numerical simulation for traffic flow and safety with cellular automata. According to the results of numerical simulations, in intelligent systems, traffic on the highway still meet the basic traffic flow equations, and the safety of the vehicle is somewhat elevated. It can be concluded that traffic flow remains unchanged, while the safety of the traffic increases in intelligent systems. And the intelligent transportation system can determine the vehicle's pitch, overtaking conditions, speed control.

\section{ACKNOWLEDGMENT}

The project was supported by Xuzhou Engineering College Students' Innovation and Entrepreneurship Fund (2014203).

\section{REFERENCES}

[1] J. Q. Xu, “Traffic engineering general,” Beijing: People's Communications Press, 1996.

[2] M. H. Lighthill, G. B. Whilham, “On kinematics waves-II: a theory of traffic flow on long crowded roads,” Proceedings, Royal Society, London, A229, pp. 317-345, 1955.

[3] L. Q. Xong, "Traffic flow theory and its application in Freeway," Wuhan University of Technology, 2003.

[4] X. P. Qiu, D. Yu, R. X. Sun, D. Yang, "Cellular automaton model based on safety distance," Journal of transportation systems engineering and information technology, vol.15(2), pp.54-60,2015.

[5] H. R. Zheng, W. C. Zhong, X. F. Ma, “Cellular traffic machine and traffic flow model considering driving angry,” Journal of Wuhan University of Technology (Transportation Science \& Engineering), vol.37(3), pp.617-621, 2013.

[6] X. Y. Zhao, "Highway traffic safety assessment based on multi-source heterogeneous data method,” Harbin :Harbin Institute of Technology, 2013.

[7] Y. Yang, "Research of performance of highway traffic safety based on operating speed,” Hunan: Hunan University, 2012.

[8] X. Z. Shen, "Research Agent-based of intelligent traffic control," Daqing Petroleum Institute, 2006.

[9] J. Zeng, H. Y. Huang, J. P. Zhang, "Considering the effects of overtaking fluid dynamic traffic flow model," Journal of GuangZhou University, vol.12(5), pp.45-48,2013.

[10] Y. Zhao, H. Y. Yao, D. M. Yan, D. H. Long, “The research of highway traffic safety evaluation,” Journal of Chongqing University, vol.31(4), pp.847-850, 2012.

[11] Z. F. Mo, "Microscopic traffic simulation study based on cellular automata,” Chongqing :Chongqing University, 2004.

[12] J. C. Zhou, Y. L. Chang, M. Guo, G. H. Wang, "Highway safety evaluation based on traffic conflict technique," Chongqing Jiaotong University (Natural Science), vol.30(5), pp.974-977,2011. 